\title{
Case series of adults with Moyamoya-related stroke and rehabilitation outcomes.
}

\author{
Robert Perna*, Joanne McGee \\ The Institute of Rehabilitation Research, Houston, Texas, USA
}

\begin{abstract}
Moyamoya disease is a rare cerebrovascular syndrome characterized by progressive stenosis and occlusion of the internal carotid arteries and other circle of Willis blood vessels. There is a growing body of research on Moyamoya, but very little describing clinical and functional outcome post rehabilitation treatment. This case series describes two individuals with Moyamoya who were in their 30's, college education, high functioning, and healthy and without any neurological history until they suffered acute intraventricular hemorrhages in physical and cognitive impairments. Both individuals showed impairments in short-term memory and higher level attention including alternating attention. Both received transdisciplinary treatment for 3 to 4 months, showed cognitive and physical recovery, and were able to return to successfully their careers. Based on these cases it appears reasonable to assume that despite the anomalous blood vessel development, some individual with this disorder may have very good stroke recoveries, though it may need follow up due to possible risk of needing a surgical intervention.
\end{abstract}

Keywords: Rehabilitation, Surgical intervention, Stroke, Hypoperfusion.

Accepted on August 24, 2017

\section{Introduction}

Moyamoya disease is a rare cerebrovascular syndrome characterized by progressive stenosis and occlusion of the internal carotid and the arteries involved in the circle of Willis, resulting in the formation of extensive small collaterals forming a network of fragile vessels in the lenticulostriate distribution. The many small collateral vessels exhibit a cloudy angiographic appearance which can look like a puff of smoke. Hence, the Japanese term "moyamoya" which means "puff of smoke." Moyamoya syndrome is uncommon outside Asia, and women are somewhat more vulnerable, with a female: male ratio of $3: 2[1,2]$. The age of diagnosis is distributed bimodally, with the highest incidence occurring in the first decade of life and another peak occurring in the third or fourth decade [2]. The etiology is uncertain with existing theories for both congenital and acquired causes. Clinical features vary dramatically as individuals may be completely asymptomatic, or they may present with intracranial hemorrhage.

The degree and localization of vascular stenosis and occlusions and the functionality of the collateral circulation determine the clinical presentation and course. Hypoperfusion and resultant neurologic or cognitive dysfunction become manifest because of collateral vessel failure [3]. Diagnosis is often based on clinical and radiographic findings including stenosis of the internal carotid arteries in conjunction with abundant collateral development [4].

Moyamoya angiographic appearance has been classified into six progressive stages [5]. The first stage is characterized by carotid stenosis without collateral vessels. The second stage involves seeing basal collateral vessels. In the third stage, these vessels become more prominent, often associated clinically with symptomatic presentations. During the fourth stage, the entire circle of Willis and posterior cerebral arteries become severely stenotic or completely occluded, Moyamoya vessels begin to narrow, and extra cranial collateral networks begin to form. It is the prominence of these extra cranial vessels which is highlight the fifth stage, and they may become the only source of blood supply to the brain.

Adult patients often present with cerebral hemorrhages and this leads to the diagnosis [4].

Treatment for Moyamoya is directed at improving collateral flow and reducing the risk of subsequent vascular events and many different techniques have been described [4]. The complex patterns of collateral blood flow may evade standard diagnostic approaches with structural imaging, though perfusion studies may confirm clinical observations. Research is unclear regarding the neuropsychological impairments that can be attributed to the Moyamoya disease itself, as opposed to secondary ischemia or hemorrhages that occur due to Moyamoya [6].

Neuropsychological effects vary greatly, but may be profound. Treatment is aimed at surgical revascularization and one revascularization technique involves dissecting and rerouting a branch of the superficial temporal artery (STA) to a distal branch of the MCA.

There has been limited neuropsychological research on Moyamoya disease in adults. The current research literature has minimal data on functional and cognitive outcomes. The purpose of this current case series is to review cases of adults who have had strokes secondary to Moyamoya and describe the physical and cognitive symptoms, course of recovery and the clinical outcomes. 


\section{Case Study}

\section{Clinical cases}

Ms. G - Ms. G is a 40 year old married woman, with a BS Degree in Business and had a 7 year history as a manager of an insurance company. She was healthy, had three children, worked fulltime, and had an active lifestyle prior to her stroke. Ms. G suffered a right hemisphere interventricular hemorrhage, resulting increased intracranial pressure, and required a VP shunt. After multiple brain MRI's, an MRA, and neurology and neurosurgery consultations she was diagnosed with Moyamoya. She suffered left side hemiparesis and impairments in various aspects of her attention skills and short-term memory. She had no significant medical history other than hypertension and had no psychiatric, substance abuse, or neurological history. There was no family history of early stroke.

After three weeks of inpatient treatment, Ms. G was evaluated for outpatient transdisciplinary post-acute brain injury rehabilitation. On the outpatient admission assessment, Ms. G had mild to moderate impairments on measures involving verbal and visual memory skills, and mathematics, and had a severe impairment on a measure involving alternating attention skills (Table 1). She initially denied any symptoms of depression on the BDI-II, but at times during treatment had become tearful and reportedly was worried that she might not be able to return to her career. She also denied anxiety, but early in treatment was somewhat avoidant about working on some of her impairments, particularly her impaired math skills. She worked diligently in treatment and on her home exercise program. She made clear and continues progress on cognitive exercises and work simulations. Cognitive retesting was not completed though work related skills improved significantly. She completed 4 months of treatment and was able to return to her previous job in a part-time manner as she was being discharged from treatment. A neurosurgical consultation at the time of discharge recommended a surgical bypass procedure to reduce the risk of stroke.

Mr. T - Mr. T was a 35 year old male with BS degree in mechanical engineering and working for an engineering company. He was generally healthy and lived with his wife of 8 years. Medical history was unremarkable until 1 year ago when he presented to the hospital with sudden onset of difficulty walking and confusion. CT of the head was significant for a large rightsided intraventricular hemorrhage with left midline shift and obstructive hydrocephalus. Follow up brain MRI's, and MRA and neurosurgery consultations resulted in a Moyamoya diagnosis. This was confirmed after seeking a second opinion. He was found to have proximal arteriopathy with distal collateralization and spontaneous rupture consistent with Moyamoya disease. $\mathrm{Mr}$. $\mathrm{T}$ was admitted for comprehensive inpatient rehabilitation after two weeks. Results of neuropsychological testing, conducted 25 days post-injury, may be referenced in Table 1. Overall, problems were identified in the areas of attention, list learning, visual memory, sequencing/cognitive flexibility, and verbal fluency. Additionally, there was evidence of problems adjusting emotionally to physical and cognitive limitations. Mr. T reported feeling sad, anxious and worried about his future. It should be noted that executive functioning/novel problem solving, insight,

Table 1. Measures involving alternating attention skills of the patients.

\begin{tabular}{|c|c|c|c|}
\hline \multirow[t]{2}{*}{ Test/Domain } & \multicolumn{2}{|c|}{$\begin{array}{c}\text { Intake } \\
\text { Standard Score (Percentile) }\end{array}$} & \multirow{2}{*}{$\begin{array}{c}\text { Discharge } \\
\text { Standard Score (Percentile) } \\
\text { Mr. T }\end{array}$} \\
\hline & Ms. G & Mr. T & \\
\hline Immediate Memory Index & --- & $76(5)$ & $97(42)$ \\
\hline List Learning & $80(9)$ & $70(2)$ & $90(25)$ \\
\hline Story Memory & --- & $85(16)$ & $105(63)$ \\
\hline Visuo-spatial/Constructional Index & --- & $84(14)$ & --- \\
\hline Figure Copy & $90(25)$ & $80(9)$ & $95(37)$ \\
\hline Judgment of Line Orientation & --- & $26-50 \%$ percentile & --- \\
\hline Language Index & --- & $78(7)$ & --- \\
\hline Naming & --- & $51-75 \%$ ile & --- \\
\hline Semantic Fluency & --- & $60(.4)$ & --- \\
\hline Attention Index & --- & $60(.4)$ & --- \\
\hline Digit Span (RBANS) & $105(63)$ & $75(5)$ & $90(25)$ \\
\hline Digit Span (WAIS-4) & $100(50)$ & --- & --- \\
\hline Coding & --- & $65(1)$ & $85(16)$ \\
\hline Delayed Memory Index & --- & $80(9)$ & --- \\
\hline List Recall & $70(2)$ & $<2 \%$ percentile & $26-50 \%$ percentile \\
\hline List Recognition & $80(9)$ & $10-16 \%$ percentile & $51-75 \%$ percentile \\
\hline Story Recall & --- & $85(16)$ & $90(25)$ \\
\hline Figure Recall & $75(5)$ & $80(9)$ & $95(37)$ \\
\hline Trail making Test-Form A & $101(53)$ & $78(7)$ & $101(53)$ \\
\hline Trail making Test-Form B & $55(<55)$ & $50(<.1)$ & $117(87)$ \\
\hline Test of Premorbid Functioning & --- & $97(42)$ & --- \\
\hline WCST-64 Categories Achieved & --- & $4\left(^{\left(>16^{\text {th }}\right.}\right.$ percentile $)$ & --- \\
\hline WCST-64 \# Perseverations & --- & 13 & --- \\
\hline WRAT-4 Arithmetic & $74(4)$ & --- & --- \\
\hline Beck Depression Inventory II & 3 & --- & --- \\
\hline
\end{tabular}


visuomotor/constructional ability and visual memory were generally preserved. After a week of inpatient rehabilitation, Mr. T was discharged home and admitted to an outpatient transdisciplinary post-acute brain injury rehabilitation program. At the time of discharge from the hospital, he was ambulatory with modified independence for uneven surfaces and transfers, independent with ADLs and modified independence for problem solving, memory and communication. Cognitive goals for outpatient rehabilitation targeted memory, attention skills, speed of processing and emotional adjustment. Mr. T's goals also included return to work.

Mr. T underwent a right craniotomy for right superficial facial temporal artery (STA) to right middle cerebral artery (MCA) bypass at 4 months post stroke. About eight months after the stroke, Mr. T was doing well neurologically after right STAMCA bypass. He no longer had cerebral perfusion deficits based on SPECT scan.

\section{Results}

This case series described two adults with Moyamoya who were both high functioning and completely asymptomatic until they each suffered acute nontraumatic right hemisphere intraventricular hemorrhages involving increase intracranial pressure and/or midline shift, and residual motor and cognitive symptoms. Both individuals exhibited impairments in shortterm memory skills and alternating attention, and this is generally consistent with other Moyamoya research [6].

Both individuals were involved in post-acute transdisciplinary treatment three days each week for 3 months. Each received occupational therapy, physical therapy, speech therapy, and neuropsychology, and groups that were run by each discipline. The treatment regime was approximately 10 skilled hours of therapy per week and each individual was given a home exercise program.

Both cases reviewed in this manuscript were acutely impaired in terms of both physical and cognitive functioning. Both individuals worked diligently in transdisciplinary rehabilitation, exhibited improved cognitive and physical functioning. Neuropsychological retesting is not fully complete, but the retesting that was done clearly shows significant improvements in various cognitive skills. Though it was initially unclear if these individuals would be able to return to their jobs, both ultimately were able to return to their jobs approximately 6-7 months post stroke.

\section{Discussion}

Most of the published Moyamoya literature discussed cases involving vessel occlusions, whereas this case series discusses individuals who suffered IVH's, but were considered high risk for occlusions. The published research on Moyamoya provides information regarding surgical options and outcomes, but very little data about rehabilitation outcomes and return to work. This situation is likely the result of both the rare nature of these cases and also the fact that cases may differ dramatically in prognostic variables. As a result it may be challenging for clinicians to offer prognoses for these cases.

\section{Conclusion}

Their rehabilitation recoveries would be considered very well given their right hemisphere IVH. However when considering prognostic variables it is important to consider that these individuals had early stroke care and interventions, was both relatively young and high functioning prior to their strokes, had a high level of motivation, and good family support. Rehabilitation clinicians working with this population may want to keep in mind the issues of these patients worrying about subsequent stroke risk, having to deal with the stress of possible subsequent bypass surgeries.

\section{References}

1. Chiu D, Shedden P, Bratina P, et al. Clinical features of Moyamoya disease in the United States. Stroke. 1998;29:1347-51.

2. Suzuki J, Kodama N. Moyamoya disease: A review. Stroke. 1983;14:104-109.

3. Jefferson A, Glosser L, Detre G, et al. Neuropsychological and perfusion MR Imaging correlates of revascularization in a case of Moyamoya syndrome. Am J Neuroradiol. 2006;27:98-100.

4. Smith ER, Scott MR. Surgical management of Moyamoya syndrome. Skull Base. 2005;15:15-26.

5. Suzuki J, Takaku A. Cerebrovascular moyamoya disease: Disease showing abnormal net-like vessels in base of brain. Arch Neurol. 1969;20:288-299.

6. Karzmark P, Zeifer PD, Tan S, et al. Effect of Moyamoya disease on neuropsychological functioning in adults. Neurosurgery. 2008;62:1048-1052.

\section{*Correspondence to:}

Robert Perna

The Institute of Rehabilitation Research

Houston,

Texas 77021, USA

Tel: 706-750-2572

E-mail:dr.perna@juno.com 